\title{
Le valutazioni economiche in Italia: una revisione sistematica della letteratura esistente
}

\author{
L. Garattini, R. Clemente, P. De Compadri, C. Denti ${ }^{*}$
}

\begin{abstract}
Aim of this paper is to provide some systematic information about Economic Evaluation studies published on national and international journals by Italian authors. All studies are in Italian or English language and published between 1994 and 2000.

The main source is the CESAV database, according to these keywords: "Economic Evaluation", "CostBenefit Analysis", "Cost-Efficacy Analysis", "Cost-Utility Analysis", Cost of Illness" and "Italy".

The study investigated the following variables: methods of analysis, illness, kind of therapy, source of data on efficacy, modelling, source of data on health resources, source of data on unit cost, type of costs, sensitivity analysis.

A comparison with the main international reviews ("The U .K. NHS Economic Evaluation Database" and "The Health Economic Evaluation Database") showed that the approach to the Economic Evaluation in Italy is consistent to the European model, even though the number of studies is still less than in other States.
\end{abstract}

\section{INTRODUZIONE}

L'allungamento della durata media della vita e il conseguente aumento di patologie croniche e degenerative, contestualmente al progresso tecnologico applicato in campo medico che rende disponibili nuove terapie sempre più costose, hanno determinato un continuo aumento della domanda di servizi sanitari in tutti i paesi sviluppati.

D'altro canto, la scarsità di risorse che caratterizza un po' tutti i sistemi sanitari rende sempre più difficile realizzare tutti i progetti per cui esiste una richiesta; di conseguenza, sarà sempre più probabile assistere all'introduzione di forme di razionamento, più o meno esplicite, destinate a lasciare insoddisfatti molti bisogni percepiti (1).

L'aumento della domanda di servizi sanitari e delle spese conseguenti, insieme all'impossibilità di soddisfare tutti i bisogni, fanno sorgere due esigenze concettualmente corre late: evitare gli sprechi e aumentare l'efficienza.

A soddisfare queste esigenze dovrebbero contribuire le Valutazioni Economiche (VE), cioè le tecniche elaborate dall'economia per razionalizzare l'allocazione e l'utilizzo di risorse scarse. In campo sanitario, infatti, le "leggi di mercato" difficilmente funzionano in modo spontaneo, a causa delle numerose peculiarità e imperfezioni che impediscono alla variabile prezzo di esercitare quell'azione "naturale" di razionamento, in grado di garantire un'efficiente allocazione delle risorse (2).

Due sono le caratteristiche peculiari che permettono di identificare un'analisi economica: in primo luogo, una VE considera sia le risorse impiegate (costi) sia i risultati prodotti dalle attività (conseguenze); in secondo luogo, una VE contribuisce a definire la scelta fra alternative di spesa. Di conseguenza, caratteristiche

CESAV (Centro di Economia Sanitaria A. e A. Valenti) dell'Istituto per le Ricerche Farmacologiche M. Negri 
peculiari delle VE sono quelle di identificare, misurare, valorizzare e confrontare i costi e le conseguenze delle alternative di carattere diagnostico-terapeutico prese in considerazione. Peraltro, accanto alle VE in senso stretto, riconducibili alle tecniche di Analisi Costo Efficacia (ACE), Costo Utilità (ACU) e Costo Beneficio (ACB), esistono VE in senso più ampio che si ricollegano principalmente alla tecnica di Cost Of Illness (COI); quest'ultima, avendo come obiettivo la quantificazione del costo di una patologia $\mathrm{o}$, più semplicemente, di un evento clinico, non prevede per definizione il confronto di alternative. A dispetto di questo limite, si tratta di analisi osservazionali in continua espansione, a causa dell'obiettiva difficoltà, in molte situazioni reali, di reperire informazioni significative in termini di costi e conseguenze delle singole alternative.

Negli ultimi anni le VE in sanità sono diventate sempre più numerose e, per questo motivo, è maturata l'esigenza di effettuare revisioni sistematiche della letteratura. A livello internazionale, sono state condotte, ad oggi, due reviews sistematiche delle VE pubblicate in lingua inglese, partendo dall'interrogazione di due database dedicati interamente alle stesse:

1. il "U .K. NHS Economic Evaluation Database (EED)" dell'università di York, nato nel 1995 e supportato dal Servizio Sanitario Inglese per individuare gli articoli sulle VE e renderli accessibili, attraverso un database pubblico, a tutti i potenziali utilizzatori;

2. il "Health Economic Evaluations Database (HEED)" dell'Office of Health Economics, un centro studi finanziato dall'associazione inglese dell'industria farmaceutica; si tratta di un servizio privato a pagamento, contenente degli abstracts che permettono di confrontare i lavori in base a uno schema comune.

Queste esperienze internazionali e le pubblicazioni conseguenti $(3,4)$, hanno stimolato il nostro interesse a condurre una ricerca bibliografica similare che avesse come oggetto le VE condotte in Italia.

La ricerca degli articoli presi in considerazione nella review è stata condotta partendo dalla banca dati della biblioteca del CESAV (il Centro di Economia Sanitaria A. Valenti dell'Istituto Mario Negri); la biblioteca, nata nel 1992, è dedicata esclusivamente alle pubblicazioni nel settore dell'economia sanitaria.

L'obiettivo principale di questo lavoro è stato, quindi, quello di fornire alcune informazioni sistematiche sugli studi di VE condotti da autori italiani, al fine di trarre alcuni spunti in materia anche alla luce dei confronti effettuati con i risultati degli studi internazionali appena citati.

\section{MATERIALI E METODI}

Al fine di comprendere lo "stato dell'arte" delle VE in Italia, è stata condotta una revisione sistematica della letteratura. A tale proposito, è stata consultata la banca dati interna del CESAV. Le parole chiave utilizzate sono state "Valutazione economica", "Analisi Costo-Beneficio", "Analisi Costo-Efficacia", "Analisi CostoUtilità", "Costo malattia" e "Italia".

Sono stati selezionati tutti gli studi originali pubblicati da autori italiani (in lingua italiana o inglese) su riviste (nazionali e internazionali) nel periodo 1994-2000.

Lo schema di analisi adottato ha indagato le seguenti variabili:

1. Tecnica di analisi: sono state prese in considerazione tutte le tipologie di VE, ivi inclusi gli studi di COI.

2. Patologia di riferimento: le patologie considerate dai diversi lavori sono state classificate in sette gruppi (patologie infettive, neurologiche, cardiovascolari, respiratorie, neoplastiche, diabete e altro) per rendere più sintetica l'analisi.

3. Tipo di intervento: questa variabile, per cui sono state individuate ex post tre categorie (terapia farmacologia, prevenzione, altro), è stata presa in considerazione solo per le VE in senso stretto, poiché le COI, indagando il costo complessivo di una patologia, non si concentrano per definizione su nessun trattamento particolare. 
4. Fonte efficacia: anche questa variabile è stata analizzata solamente all'interno delle VE in senso stretto, essendo per definizione esclusa dalle COI (1). Le categorie considerate sono state le seguenti: metanalisi, registri sanitari (dati derivati da ospedali, ASL o altre strutture sanitarie) e sperimentazioni cliniche.

5. Albero decisionale: anche in questo caso, come nel successivo, si è indagata la presenza o meno di questo strumento nelle VE in senso stretto (le sole per cui ha senso utilizzarlo).

6. Modelling: dopo aver osservato in quali VE in senso stretto sono stati utilizzati i modelli, si è indagato il tipo di modello usato.

7. Fonte risorse sanitarie: è stata indagata in tutti i lavori e le categorie considerate sono state le seguenti: registri sanitari, sperimentazioni cliniche, dati sperimentali, dati di letteratura (cioè quelli derivati da lavori già pubblicati).

8. Tipologia di costi: questa informazione è stata raccolta per tutti i lavori e ha indagato quali VE hanno quantificato i costi classificati secondo la tripartizione classica (diretti, indiretti e intangibili).

9. Fonte costo unitario: questa informazione è stata raccolta solo per la sottocategoria dei costi ospedali eri, i più rilevanti in sanità, con l'obiettivo di verificare quali lavori abbiano analizzato, in aggiunta alle tariffe in vigore nel SSN, i costi reali delle strutture ospedaliere.

10. Analisi di sensibilità: per questa variabile ci si è limitati a verificare se gli studi selezionati abbiano o meno effettuato tale tipo di analisi, giudicata parte integrante della metodologia delle VE (2).

Due ricercatori hanno eseguito, separatamente, l'analisi di ogni articolo selezionato, al fine di validare la consistenza delle classificazioni effettuate (molte voci sono state decise ex post) e, quindi, la presentazione dei risultati della review stessa.

\section{RISULTATI}

Dei circa 2000 lavori originariamente selezionati sono stati eliminati, in primo luogo, tutti quelli che non erano articoli; all'interno di questi ultimi, sono stati esclusi quelli che corrispondevano a articoli pubblicati in un periodo diverso da quello analizzato (1994-2000) e firmati da autori stranieri, nonché tutti quelli non contenenti studi originali (editoriali, articoli di metodologia, reviews, ecc.); in questo modo, si è arrivati a selezionare 41 articoli che soddisfacevano i criteri di selezione illustrati nel paragrafo precedente (Tabella 1). Fra questi, 16 sono, rispettivamente, ACE e COI; 2 sono ACB e ACU; 2 lavori, inoltre, hanno associato ACE e ACB e 3 ACE e ACU (Figura 1).

Nove articoli riguardano le patologie infettive, 5 le neoplasie, 4 le patologie neurologiche, quelle cardiovascolari, il diabete e le patologie respiratorie; i rimanenti Il si sono occupati di patologie varie (Figura 2).

Per quanto riguarda il tipo di intervento, $17 \mathrm{VE}$ in senso stretto vertono sulla terapia farmacologica, $5 \mathrm{si}$ concentrano su strategie di prevenzione, mentre 3 si occupano di temi vari (procedure diagnostiche e percorsi terapeutici).

Dodici articoli di VE in senso stretto hanno basato la dimostrazione di efficacia su sperimentazioni cliniche, 10 su registri sanitari e 3 su metanalisi (Figura3).

La presenza di alberi decisionali è stata riscontrata in 5 studi di VE in senso stretto, mentre ben 20 non li hanno utilizzati.

Quasi la metà dei lavori [precisamente 17] ha prodotto dati sperimentali per stimare le risorse sanitarie, mentre 10 sono ricorsi ai registri sanitari, 10 a dati già presenti in letteratura e 4 a dati ricavati da sperimentazioni cliniche (Figura 4). 


\begin{tabular}{|c|c|c|c|c|}
\hline Autori & Titelo articole & $\begin{array}{c}\text { Tipo } \\
\text { Analisi }\end{array}$ & Rivista & $\begin{array}{c}\text { Anno di } \\
\text { pubblicazione }\end{array}$ \\
\hline $\begin{array}{l}\text { Cavallo M.. } \\
\text { Sassi } F_{\text {. }} \\
\text { Geraci P. }\end{array}$ & $\begin{array}{l}\text { Anahisi costo utilita della terapia } \\
\text { intensiva nel trattanento delficas } \\
\text { emorragico }\end{array}$ & $\mathrm{ACU}$ & MECOSAN & 1994 \\
\hline $\begin{array}{l}\text { Derricheli V., } \\
\text { Casadiso G. } \\
\text { Lancioti G., } \\
\text { Novaco F., } \\
\text { Olvier Jeferson T. }\end{array}$ & $\begin{array}{l}\text { The Emilia costing study: } \\
\text { valutazione e co nomica } \\
\text { dell'impatto economico della } \\
\text { salmonellosi umena }\end{array}$ & COI & MECOSAN & 1994 \\
\hline Fedele D. & Neuropatia diabetica: $\mathrm{i}$ costi & $\mathrm{COI}$ & II Diabete & 1994 \\
\hline $\begin{array}{l}\text { Mapelt V, } \\
\text { Schuleaburg G. } \\
\text { Larsct U." } \\
\text { Alloff P. Rossi. F, }\end{array}$ & $\begin{array}{l}\text { Economic Evaluation of } \\
\text { Lenograstim (glycosybted rHuG- } \\
\text { C SF) in the Treatment of } \\
\text { Inflammatory Breast Cancer for } \\
\text { Germany and ltaly. }\end{array}$ & $A C E$ & $\begin{array}{l}\text { Pharmace } \\
\text { Eccoonics }\end{array}$ & 1994 \\
\hline Tamicone R. & $\begin{array}{l}\text { Vahutare il costo di ura malattia. II } \\
\text { costo de Ifischemia critica cronica } \\
\text { degli arti. }\end{array}$ & $\mathrm{COI}$ & FarmecoEcorkmisi & 1994 \\
\hline $\begin{array}{l}\text { Dal Negro R., } \\
\text { Tirco P., } \\
\text { Micheletto C, } \\
\text { Poman C. }\end{array}$ & I costi sociali de Rasma hronchinle & $\mathrm{COl}$ & $\begin{array}{l}\text { Fartaco } \\
\text { Economia }\end{array}$ & 1995 \\
\hline Farrore $\mathrm{G}$ & $\begin{array}{l}\text { Analisi costo-effieacia del } \\
\text { trattamento e della prevencione } \\
\text { con aspirina in pazienti con } \\
\text { sospetto infarto niocardion acuto } \\
\text { e ad alto rischio di incidenti } \\
\text { vascolari }\end{array}$ & $\mathrm{ACE}$ & MECOSAN & 1995 \\
\hline $\begin{array}{l}\text { Garattini } L \text {, } \\
\text { Canelli T., } \\
\text { Tribbia G. } \\
\text { Scopeltiti D. }\end{array}$ & $\begin{array}{l}\text { Economic Evaluation of an } \\
\text { Edacational Campaign for Early } \\
\text { Diagnosis of Cutaneous } \\
\text { Melanoma. }\end{array}$ & ACE & $\begin{array}{l}\text { Pharmace } \\
\text { Ecconornics }\end{array}$ & 1996 \\
\hline $\begin{array}{l}\text { Maniovani L.. } \\
\text { Montanell R., } \\
\text { Tanricone R. }\end{array}$ & $\begin{array}{l}\text { Valusazione economica dell'use di } \\
\text { gas plasma di perossido di } \\
\text { ifrogeno nella sterilozazione in } \\
\text { ambito ospedaliero }\end{array}$ & $A C B$ & MECOSAN & 1996 \\
\hline $\begin{array}{l}\text { Neri M., } \\
\text { Miglori G.B., } \\
\text { Spanevello A., } \\
\text { Berra D, } \\
\text { Nicolin E et al }\end{array}$ & $\begin{array}{l}\text { Economic an alysis of two } \\
\text { structured treatment and teaching } \\
\text { programs on asthma }\end{array}$ & $\begin{array}{l}\mathrm{ACB} \\
\mathrm{ACE}\end{array}$ & Alkrgy & 1996 \\
\hline $\begin{array}{l}\text { Wallari P., } \\
\text { Fattore, G., } \\
\text { Siegel J., Paltiel } \\
\text { A., Weislein M. }\end{array}$ & $\begin{array}{l}\text { Economic evaluation of HIV } \\
\text { testing among intravenous drug } \\
\text { users }\end{array}$ & ACE & $\begin{array}{l}\text { Intemational Joumal } \\
\text { of Teclunology } \\
\text { Assessmont in } \\
\text { Health Care }\end{array}$ & 1996 \\
\hline $\begin{array}{l}\text { Garattini L. } \\
\text { Barosi M, } \\
\text { Brunetti M., } \\
\text { Sakioni F. }\end{array}$ & $\begin{array}{l}\text { Economic Evaluation of ACE } \\
\text { Inhibitor Treatm ent of } \\
\text { Nephropatby in Patients with } \\
\text { Insulin-Dependent Diabetes } \\
\text { Melinus in laly }\end{array}$ & ACE & $\begin{array}{l}\text { Phinnace } \\
\text { Economics }\end{array}$ & 1997 \\
\hline Becagh P. & $\begin{array}{l}\text { Profilo farmacoeconomico } \\
\text { dellakndronato in docne in post- } \\
\text { menopausa con fratture vertebrafi } \\
\text { preesistentr analsi costo-ntilitit di } \\
\text { tipo Hetime }\end{array}$ & $\mathrm{ACU}$ & $\begin{array}{c}\text { Gionale di } \\
\text { Farmacoeconomi }\end{array}$ & 1997 \\
\hline Berto V: & $\begin{array}{l}\text { Profilo fammacoesonomico della } \\
\text { monoterapia con zidovoxdina e } \\
\text { della terapia tripla con saquinavirt } \\
\text { zalcitabina tridovodina in pazicati } \\
\text { con infezione da HIV in stadio } \\
\text { avanzato }\end{array}$ & $\begin{array}{l}\mathrm{ACE} \\
\mathrm{ACU}\end{array}$ & $\begin{array}{l}\text { Gixmale di } \\
\text { Farmacocconomia }\end{array}$ & 1997 \\
\hline $\begin{array}{l}\text { Bonistalli L. } \\
\text { Costanimi M. }\end{array}$ & $\begin{array}{l}\text { Chemioterapii post-operatoria in } \\
\text { pazienti con cancro del colon- } \\
\text { retto: valutazione dei rapporti } \\
\text { costo-effscacia e costo-utitat }\end{array}$ & $\begin{array}{l}\mathrm{ACE} \\
\mathrm{ACU}\end{array}$ & $\begin{array}{l}\text { Giomak di } \\
\text { Farmacoeconomita }\end{array}$ & 1997 \\
\hline
\end{tabular}

Tabella 1 segue 


\begin{tabular}{|c|c|c|c|c|}
\hline Autori & Titolo articolo & $\begin{array}{c}\text { Tipo } \\
\text { Analisi }\end{array}$ & Rivista & $\begin{array}{c}\text { Anno di } \\
\text { pubblicazione }\end{array}$ \\
\hline $\begin{array}{l}\text { Cavallo M., } \\
\text { Fattore } \mathrm{G}\end{array}$ & $\begin{array}{l}\text { The economic and socis burden } \\
\text { of Atheiner Disease on families } \\
\text { in the Lombardy Region of ltaly }\end{array}$ & $\mathrm{COI}$ & $\begin{array}{l}\text { Alsciner Disease } \\
\text { and Associated } \\
\text { Disorders }\end{array}$ & 1997 \\
\hline $\begin{array}{l}\text { Liberato } \mathrm{N}_{\text {. }} \\
\text { Quaglini } \mathrm{S}_{\text {. }} \\
\text { Barcosici }\end{array}$ & $\begin{array}{l}\text { Cost effectiveness of imterferon } \\
\text { alfa in chronic myelogenous } \\
\text { leukemia }\end{array}$ & $A C E$ & $\begin{array}{l}\text { Joumal of Climical } \\
\text { Oncology }\end{array}$ & 1997 \\
\hline Tamicone $\mathrm{R}$. & $\begin{array}{l}\text { Metologia di indagine e primi } \\
\text { risultati sul costo sociale della } \\
\text { depressione maggiore in ltalia }\end{array}$ & $\mathrm{COI}$ & MECOSAN & 1997 \\
\hline Trippoli S. & $\begin{array}{l}\text { Trattamento dei pazienti con } \\
\text { mie loma multip lo di nuova } \\
\text { diagnosi: analisi dei dati di } \\
\text { sopravvivenza o valutazione } \\
\text { costo-efficacia }\end{array}$ & ACE & $\begin{array}{l}\text { Gimale di } \\
\text { Farmacoeconoms }\end{array}$ & 1997 \\
\hline $\begin{array}{l}\text { Fattore } \mathrm{G} \text {, } \\
\text { Lazoaro } \mathrm{C} \text {. }\end{array}$ & $\begin{array}{l}\text { Analisi Costi-Efficacia della } \\
\text { fertilizazione in vitro in Italia }\end{array}$ & $\mathrm{ACE}$ & MECOSAN & 1998 \\
\hline Ferrari M. & $\begin{array}{l}\text { The Economic Burden of } \\
\text { Migraine to Suciety }\end{array}$ & $\mathrm{COI}$ & $\begin{array}{l}\text { Phamrico } \\
\text { Economics }\end{array}$ & 1998 \\
\hline $\begin{array}{l}\text { Lucioni C. } \\
\text { Cipriani } \mathrm{Y}_{\text {, }} \\
\text { Mazzi S., } \\
\text { Panumzio M. }\end{array}$ & $\begin{array}{l}\text { Cost of an Outbreak of Heputitis } \\
\text { A in Pugin, Italy }\end{array}$ & $\mathrm{COI}$ & $\begin{array}{l}\text { Pharmaco } \\
\text { Econxonics }\end{array}$ & 1998 \\
\hline $\begin{array}{l}\text { Berto P., } \\
\text { Coccheri S. }\end{array}$ & $\begin{array}{l}\text { Una valutazione economica di } \\
\text { studi di profilassi e di tratamento } \\
\text { domictare delh trombosi venosa } \\
\text { profonda con eparina a basso } \\
\text { peso molocolare }\end{array}$ & $\mathrm{ACE}$ & $\begin{array}{l}\text { Giocnale di } \\
\text { Farmacoeconomia }\end{array}$ & 1999 \\
\hline $\begin{array}{l}\text { Berto P., } \\
\text { Gianfrate F. }\end{array}$ & II costo delfasma in Italia & $\mathrm{COI}$ & $\begin{array}{c}\text { Giotrak di } \\
\text { Farmacoeconomi }\end{array}$ & 1999 \\
\hline $\begin{array}{l}\text { Beutels Ph., } \\
\text { Bcoanni P., } \\
\text { Tomans } \mathrm{G} \text {. } \\
\text { Canale F., } \\
\text { Cuneo Corvari P. }\end{array}$ & $\begin{array}{l}\text { An economic evaluation of } \\
\text { universal pertussis vaccination in } \\
\text { Italy }\end{array}$ & $\begin{array}{l}\mathrm{ACE} / \\
\mathrm{ACB}\end{array}$ & Vhocine & 1999 \\
\hline $\begin{array}{l}\text { Boncanini A. } \\
\text { Loyd A.C., } \\
\text { Ganfrate F. }\end{array}$ & $\begin{array}{l}\text { Analisi di costo-efficacia di } \\
\text { flutica sone propionato e } \\
\text { budesonide nel trattameato } \\
\text { delfasma di aduli e bambini }\end{array}$ & $\mathrm{ACE}$ & $\begin{array}{l}\text { Giomale di } \\
\text { Farmecocooocmia }\end{array}$ & 1999 \\
\hline $\begin{array}{l}\text { Brusaferto S., } \\
\text { Rizzato E., } \\
\text { Damsini G }\end{array}$ & $\begin{array}{l}\text { Valutazione economica di un } \\
\text { programma per la prevenzione } \\
\text { delle infezioni delle vic uninarie da } \\
\text { cateterismo vescicale in un } \\
\text { contesto ospedaliero italsano }\end{array}$ & $\mathrm{ACB}$ & Vew \& Review & 1999 \\
\hline Cantagallo $A$. & $\begin{array}{l}\text { Costo efficacia di un programma } \\
\text { di prevenzione con dieta a basso } \\
\text { co ntenuto p rot eico nella } \\
\text { nefiopatia diabetica }\end{array}$ & $\mathrm{ACE}$ & MECOSAN & 1999 \\
\hline $\begin{array}{l}\text { Costantini M., } \\
\text { Bartolini F., } \\
\text { Bonistalli L. }\end{array}$ & $\begin{array}{l}\text { Goserelin in pazienti con cancro } \\
\text { prostatico localmente avanzato: } \\
\text { a n a lisi c os to / e ffic a c ia e } \\
\text { costo/utilita }\end{array}$ & $\begin{array}{l}\mathrm{ACE} / \\
\mathrm{ACU}\end{array}$ & $\begin{array}{l}\text { Giomale di } \\
\text { Fammacosonomia }\end{array}$ & 1999 \\
\hline $\begin{array}{l}\text { Da Villa } \mathrm{G} \text {, } \\
\text { Sepe A. }\end{array}$ & $\begin{array}{l}\text { Imrounication programme against } \\
\text { hepatitis B vinus infection in ltaly: } \\
\text { cost-effectiveness }\end{array}$ & $\mathrm{ACE}$ & Viacine & 1999 \\
\hline $\begin{array}{l}\text { Fornaro } \text { P., } \\
\text { Gandini } F_{4} \\
\text { Marin M. } \\
\text { Pedrazai C. et al. }\end{array}$ & $\begin{array}{l}\text { Epidemiology and cost analysis of } \\
\text { varicella in Italy: results of a } \\
\text { sentinel study in the pediatric } \\
\text { practice }\end{array}$ & $\mathrm{COI}$ & $\begin{array}{l}\text { Pedatric Infiections } \\
\text { Disease Joumal }\end{array}$ & 1999 \\
\hline $\begin{array}{l}\text { Gugianti A., } \\
\text { Rossetti } C_{\text {., }} \\
\text { Grimaldi } \text { A.t } \\
\text { Famo } \mathrm{E} \text {. }\end{array}$ & $\begin{array}{l}\text { Due esercizi di analisi decisionale } \\
\text { sulla introduzione della PET nei } \\
\text { noduli polmonari solitari ne llo } \\
\text { stag ing i p a zienticon } \\
\text { N.S.C.L.C. }\end{array}$ & $A C E$ & MECOSAN & 1999 \\
\hline
\end{tabular}




\begin{tabular}{|c|c|c|c|c|}
\hline Autori & Titolo articolo & $\begin{array}{c}\text { Tipo } \\
\text { Analisi }\end{array}$ & Rivista & $\begin{array}{c}\text { Anno di } \\
\text { pubblicazione }\end{array}$ \\
\hline $\begin{array}{l}\text { Lucioni C., } \\
\text { Ciriminna S., } \\
\text { Di Carlo P., } \\
\text { Mazzi S., Serra } \\
\text { G., Titone L. }\end{array}$ & $\begin{array}{l}\text { Il costo sociale del morbillo in età } \\
\text { pediatrica. L'epidemia a Palermo } \\
\text { nel 1996-97 }\end{array}$ & $\mathrm{COI}$ & $\begin{array}{l}\text { Pharmaco } \\
\text { Economics }\end{array}$ & 1999 \\
\hline $\begin{array}{l}\text { Mantovani L., } \\
\text { Belisari A., } \\
\text { Dobrilla G. }\end{array}$ & $\begin{array}{l}\text { Valutazione economica de } 1 \\
\text { lansoprazolo nel trattamento dei } \\
\text { pazienti affetti da reflusso } \\
\text { esofageo }\end{array}$ & $\mathrm{ACE}$ & $\begin{array}{c}\text { Pharmaco } \\
\text { Economics } \\
\text { edizione italiana }\end{array}$ & 1999 \\
\hline $\begin{array}{l}\text { Migliori G.B., } \\
\text { Ambrosetti M., } \\
\text { Besozzi G., } \\
\text { Farris B. et al. }\end{array}$ & $\begin{array}{l}\text { Cost comparison of different } \\
\text { management policies for } \\
\text { tubercolosis patients in Italy }\end{array}$ & $\mathrm{ACE}$ & $\begin{array}{l}\text { Bulletin of theWorld } \\
\text { Health Organization }\end{array}$ & 1999 \\
\hline $\begin{array}{l}\text { Triossi O., } \\
\text { Tamperi I., } \\
\text { Casetti T., Buda } \\
\text { S., Cuttin S., } \\
\text { Degli Espositi E. }\end{array}$ & $\begin{array}{l}\text { P r o ge t t o d i D is p e p s ia } \\
\text { (DYSPRO): creazione di una } \\
\text { linea guida di comportamento } \\
\text { diagnostico teraputico condivisa } \\
\text { dai Medici di Medicina Generale } \\
\text { e sua applicazione, valutazione } \\
\text { farmacoceonomica e analisi degli } \\
\text { esiti }\end{array}$ & $\mathrm{COI}$ & $\begin{array}{c}\text { Giornale di } \\
\text { Farmaeconomia }\end{array}$ & 1999 \\
\hline $\begin{array}{l}\text { Attanasio E., } \\
\text { Russo P. }\end{array}$ & $\begin{array}{l}\text { Analisi costo-efficacia tra due } \\
\text { statine sulla base diindicatori } \\
\text { intermedi }\end{array}$ & $\mathrm{ACE}$ & FarmacoEconomia & 2000 \\
\hline $\begin{array}{l}\text { Berto P., } \\
\text { Tinuper P., } \\
\text { Viaggi S. }\end{array}$ & $\begin{array}{l}\text { Cost of Illness of Epilepsy in Italy. } \\
\text { D a t a from a Mu lt ic e n tr e } \\
\text { Observational Study (Episcreen) }\end{array}$ & COI & $\begin{array}{l}\text { Pharmaco } \\
\text { Economics }\end{array}$ & 2000 \\
\hline $\begin{array}{l}\text { Tarricone R., } \\
\text { Gerzeli S., } \\
\text { Montanelli R., } \\
\text { Frattura L., } \\
\text { Percudani M., } \\
\text { Racagni G. }\end{array}$ & $\begin{array}{l}\text { Direct and ind irect costs of } \\
\text { schizophrenia in community } \\
\text { psychiatric services in Italy. The } \\
\text { GISIES study }\end{array}$ & OI & Health Policy & 2000 \\
\hline $\begin{array}{l}\text { Tediosi F., } \\
\text { Chiaffarino F., } \\
\text { Parazzini F., } \\
\text { Coscelli C., } \\
\text { Garattini L. }\end{array}$ & $\begin{array}{l}\text { I costi del piede diabetico in Italia: } \\
\text { dati dal progetto RECORD }\end{array}$ & $\mathrm{COI}$ & $\begin{array}{c}\text { Pharmaco } \\
\text { Economics } \\
\text { edizione italiana }\end{array}$ & 2000 \\
\hline $\begin{array}{l}\text { Tediosi F., } \\
\text { Parazzini F., } \\
\text { Bortolotti A., } \\
\text { Garattini L. }\end{array}$ & $\begin{array}{l}\text { The cost of urinary incontinence in } \\
\text { italian women }\end{array}$ & $\mathrm{COI}$ & $\begin{array}{l}\text { Pharmaco } \\
\text { Economics }\end{array}$ & 2000 \\
\hline
\end{tabular}

segue Tabella 1

I modelli sono stati applicati solo in 8 lavori di VE in senso stretto; fra questi, 3 sono modelli di Markov, uno è un modello di Gompertz, 3 sono un'integrazione fra Q-TWIST e modello di Gompertz e uno è un DEALE. Venticinque lavori hanno analizzato solo i costi diretti, 15 hanno considerato i costi sia diretti sia indiretti, 
mentre uno ha analizzato solo i costi indiretti (Figura 5). Va sottolineato che nessuno degli studi selezionati ha cercato di monetizzare la categoria dei costi intangibili.

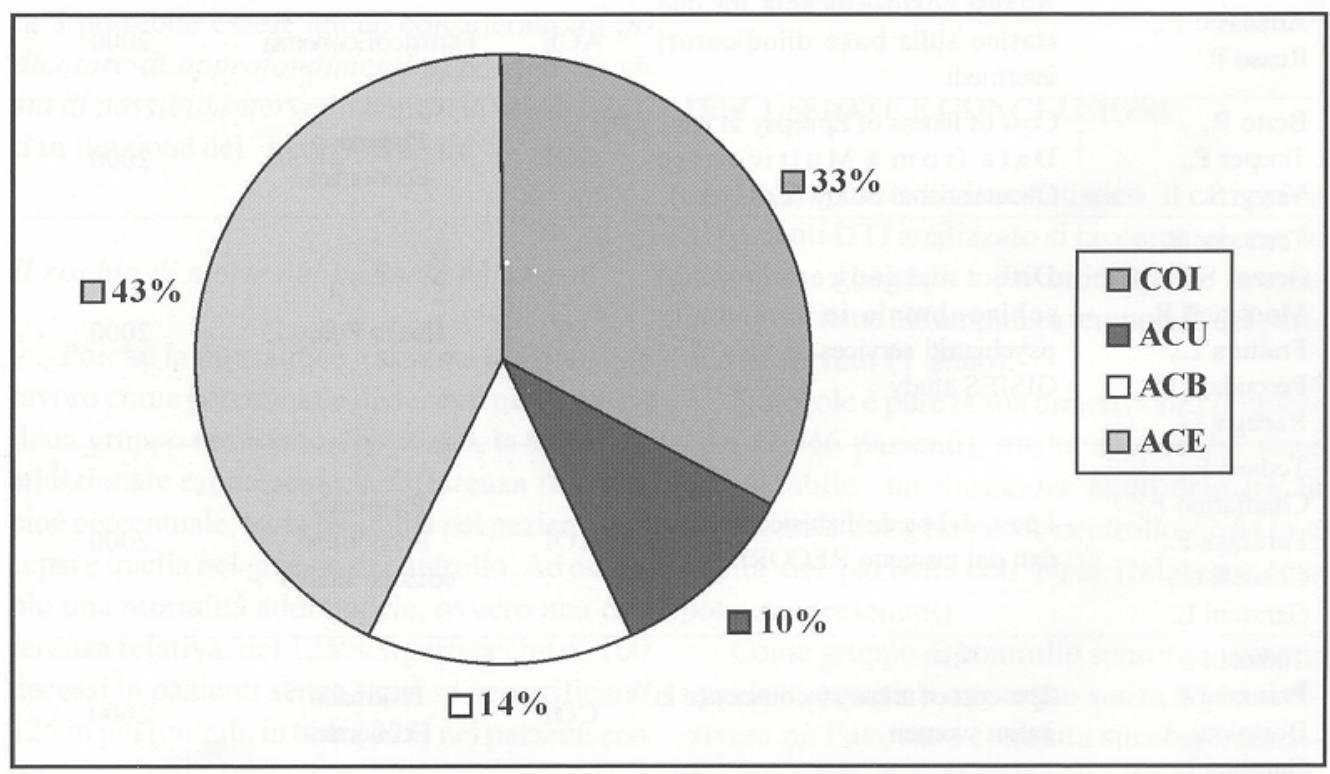

Figura 1 Tecnica di analisi.

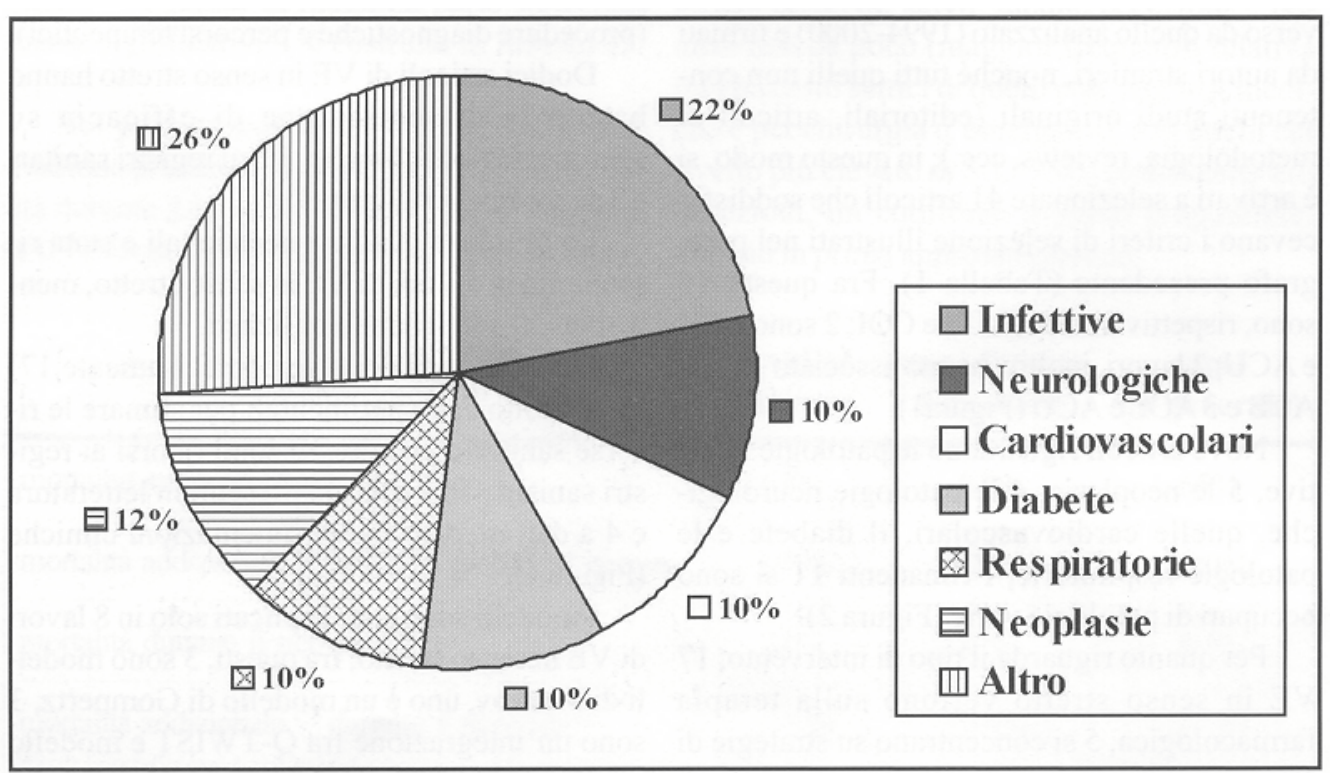

Figura 2

Patologie di riferimento nel caso di Valutazioni Economiche. 
Più di due terzi degli studi [32] hanno utilizzato essenzialmente i prezzi e le tariffe per calcolare i costi unitari ospedalieri, mentre 9 hanno elaborato dati sperimentali in ambito ospedaliero.

Infine, l'uso dell'analisi di sensibilità ha spaccato in due in modo pressoché perfetto il campione fra studi che la hanno applicata (21) e studi che non ne hanno fatto uso (20).

\section{DISCUSSIONE}

Questo paragrafo finale ha come obiettivi principali quelli di trarre alcune considerazioni generali relativamente al modo in cui vengono svolte le VE in Italia e di effettuare un confronto, laddove possibile, con le revisioni internazionali della letteratura già pubblicate $(3,4)$.

Un primo dato evidente riguarda il gap esistente fra teoria e pratica nel campo delle VE, sia per quanto riguarda le tecniche di analisi, sia rispetto alle categorie di costi indagate. Come già sottolineato, infatti, la teoria in materia restringe la definizione di VE alle tecniche di ACE, ACB, ACU; inoltre raccomanda sempre di prendere in considerazione anche i costi indiretti e intangibili (2). L'analisi evidenzia come buona parte delle VE presenti nel panorama italiano sia rappresentata da COI e vengano indagati principalmente i costi diretti, ignorando completamente quelli intangibili e interessandosi in modo limitato a quelli indiretti.

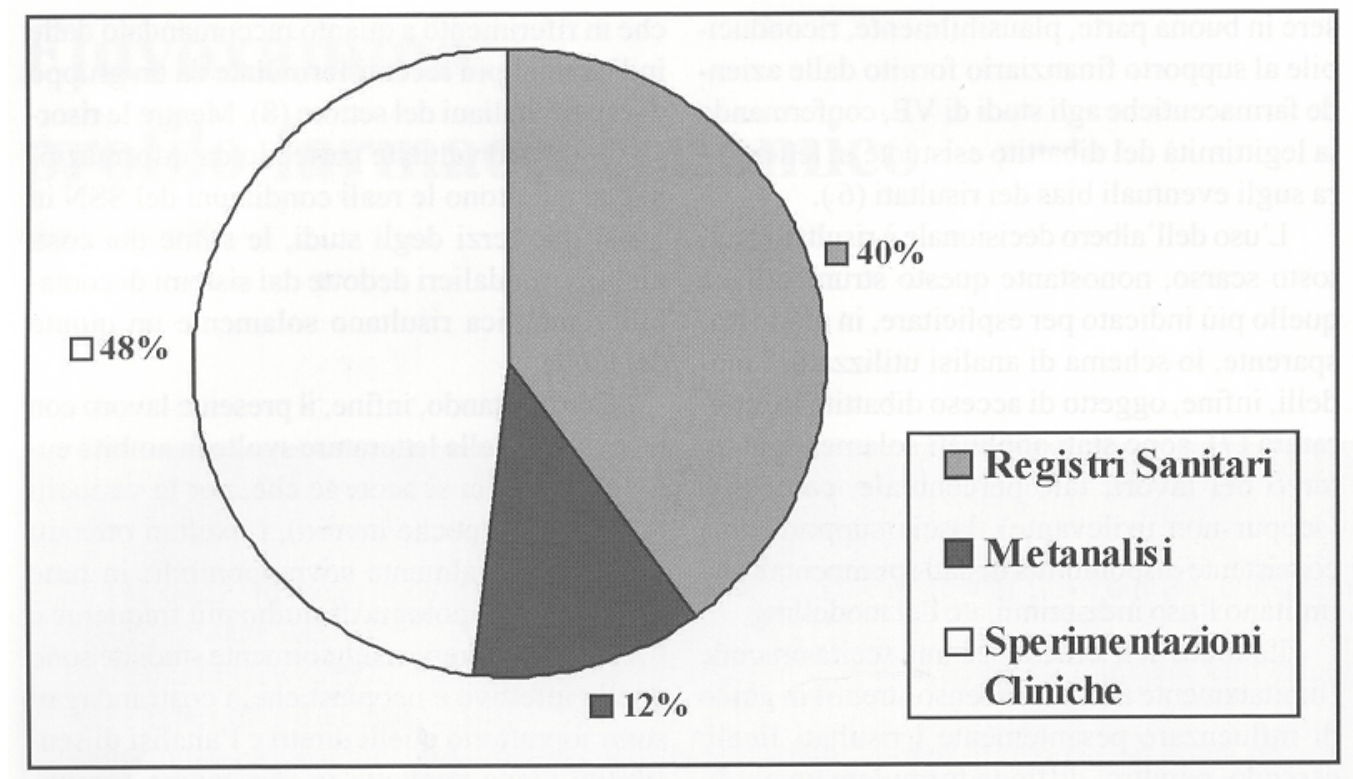

Figura 3

Fonte Efficacia delle Valutazioni Economiche in senso stretto. 


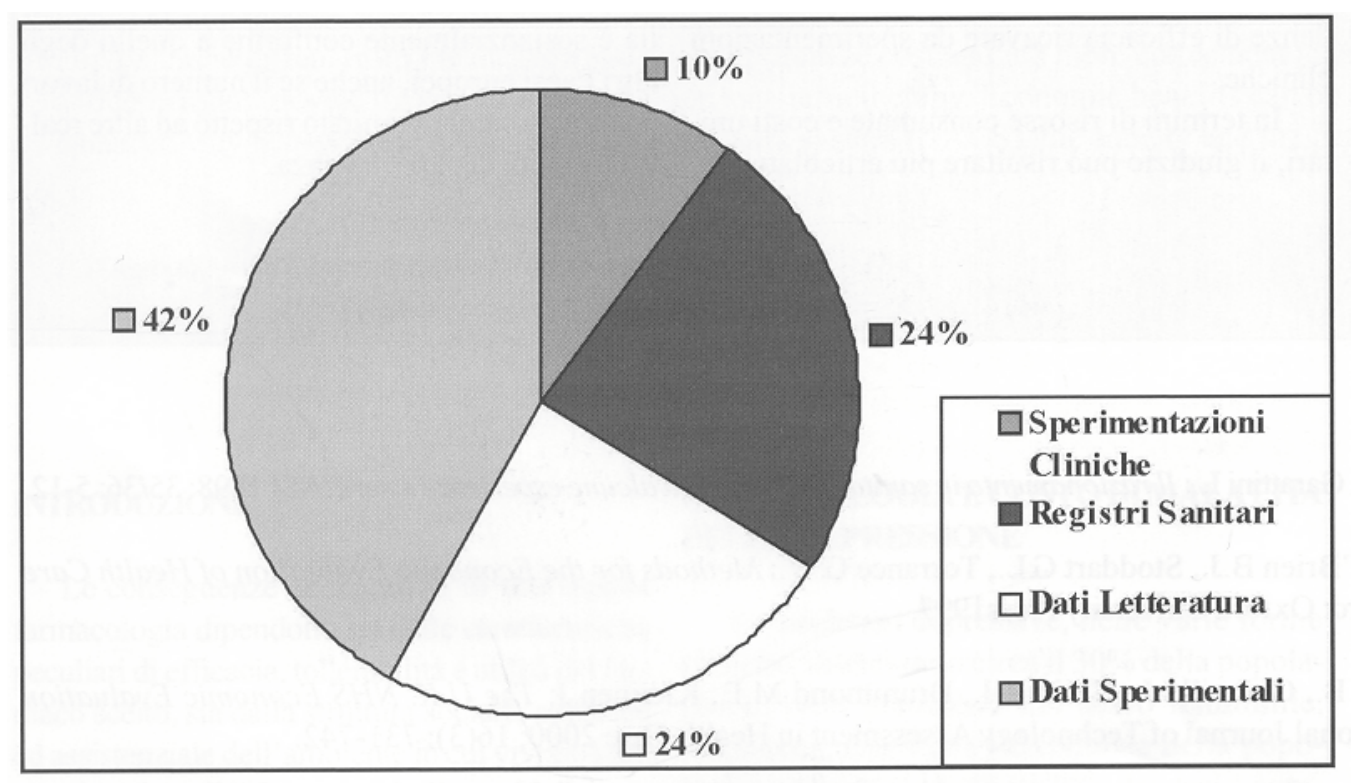

Figura 4

Fonte Risorse delle Valutazioni Economiche.

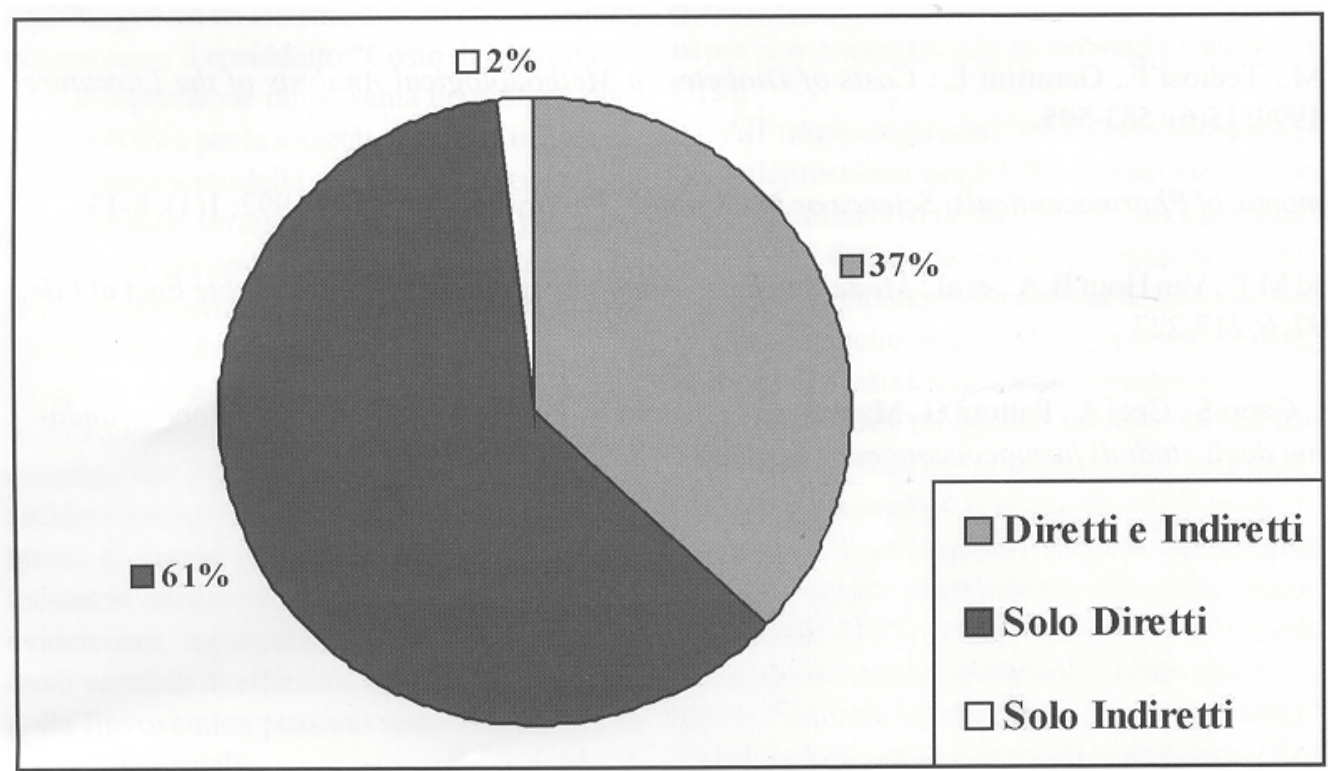

Figura 5

Tipologie di Costi. 
Più di due terzi delle VE si concentra sulla terapia farmacologica; questo risultato può essere in buona parte, plausibilmente, riconducibile al supporto finanziario fornito dalle aziende farmaceutiche agli studi di VE, confermando la legittimità del dibattito esistente in letteratura sugli eventuali bias dei risultati (6).

L'uso dell'albero decisionale è risultato piuttosto scarso, nonostante questo strumento sia quello più indicato per esplicitare, in modo trasparente, lo schema di analisi utilizzato. I modelli, infine, oggetto di acceso dibattito in letteratura (7), sono stati applicati solamente in un terzo dei lavori; tale percentuale, contenuta (seppur non irrilevante), lascia supporre una consistente disponibilità di dati sperimentali che limitano l'uso indiscriminato del modelling.

La fonte dell'efficacia è una scelta cruciale (limitatamente alle VE in senso stretto) in grado di influenzare pesantemente i risultati finali; essendo, peraltro, difficile formulare un giudizio di valore non entrando approfonditamente nel merito dei singoli studi, ci si limita a constatare che solo la metà degli studi si basa su evidenze di efficacia ricavate da sperimentazioni cliniche.

In termini di risorse consumate e costi unitari, il giudizio può risultare più articolato, anche in riferimento a quanto raccomandato dalle indicazioni più recenti formulate da un gruppo di esperti italiani del settore (8). Mentre le risorse sono state valutate partendo da informazioni che riflettono le reali condizioni del SSN in quasi due terzi degli studi, le stime dei costi unitari ospedalieri dedotte dai sistemi di contabilità analitica risultano solamente un quinto del totale.

Confrontando, infine, il presente lavoro con le revisioni della letterature svolte in ambito europeo $(3,4)$, ci si accorge che, per le variabili comparabili (poche invero), i risultati ottenuti sono sostanzialmente sovrapponibili: in tutte le reviews la tipologia di studio più frequente è l'ACE, le patologie maggiormente studiate sono quelle infettive e neoplastiche, i costi indagati sono soprattutto quelli diretti e l'analisi di sensibilità viene condotta in una buona percentuale degli studi.

La somiglianza dei risultati sembrerebbe testimoniare che il modo di condurre le VE in Italia è sostanzialmente conforme a quello degli altri Paesi europei, anche se il numero di lavori pubblicati sembra limitato rispetto ad altre realtà nazionali di pari rilevanza.

\section{BIBLIOGRAFIA}

1. Tediosi F., Fattore G., Garattini L.: Il razionamento in sanità: riflessioni su alcune esperienze estere, ASI 1998; 35/36: 5-12.

2. Drummond M.F., O'Brien B.J., Stoddart G.L., Torrance G.W.: Methods for the Economic Evaluation of Health Care Programmes, Oxford: Oxford University Press 1997.

3. Nixon J., Stoykova B., Glanville J., Christie J., Drummond M.F., K1eijnen J: The U.K. NHS Economic Evaluation Database, Intemational Journal of Technology Assessment in Health Care 2000; 16(3): 731742.

4. Pritchard C.: What Do We Know about the Economic Evaluations Literature? A review of HEED, Journal of Drug Assessment 2001; 4: 1-70.

5. Pagano E., Brunetti M., Tediosi F., Garattini L.: Costs of Diabetes: a Methodological Analysis of the Literature, PharmacoEconomics 1999; 15(6): 583-595.

6. Drummond M.F.: Economic of Pharmaceuticals. Science or Marketing?, PharmacoEconomics 1992; 1(1): 8-13.

7. Buxton M., Drummond M.F., Van Hout B.A., et al: Modelling in Economic Evaluation: an Unavoidable Fact of Life,. HealthEconomics 1997; 6: 217-227.

8. Attanasio E., Bruzzi P., Capri S., CeciA., Fattore G, Mantovani L., Merlo F., Reggio S., Terranova L.: Raccomandazioni per la conduzione degli studi di farmacoeconomia: la guida GISF, Mecosan 1999; 29 $\left(\operatorname{sez} 2^{\mathrm{a}}\right)$. 